\title{
Complex Stochastic Boolean Systems: Comparing Bitstrings with the Same Hamming Weight
}

\author{
Luis González \\ Department of Mathematics, Research Institute SIANI, University of Las Palmas de Gran Canaria, Campus de Tafira, \\ 35017 Las Palmas de Gran Canaria, Spain
}

Correspondence should be addressed to Luis González; luisglez@dma.ulpgc.es

Received 31 October 2013; Accepted 11 January 2014; Published 24 March 2014

Academic Editor: Chun-Yi Su

Copyright (C) 2014 Luis González. This is an open access article distributed under the Creative Commons Attribution License, which permits unrestricted use, distribution, and reproduction in any medium, provided the original work is properly cited.

A complex stochastic Boolean system (CSBS) is a complex system depending on an arbitrarily large number $n$ of random Boolean variables. CSBSs arise in many different areas of science and engineering. A proper mathematical model for the analysis of such systems is based on the intrinsic order: a partial order relation defined on the set $\{0,1\}^{n}$ of all binary $n$-tuples of 0 s and 1 s. The intrinsic order enables one to compare the occurrence probabilities of two given binary $n$-tuples with no need to compute them, simply looking at the relative positions of their 0s and 1s. Regarding the analysis of CSBSs, the intrinsic order reduces the complexity of the problem from exponential ( $2^{n}$ binary $n$-tuples) to linear ( $n$ Boolean variables). In this paper, using the intrinsic ordering, we compare the occurrence probabilities of any two binary $n$-tuples having the same number of 1-bits (i.e., the same Hamming weight). Our results can be applied to any CSBS with mutually independent Boolean variables.

\section{Introduction}

This paper deals with the mathematical modeling of a special kind of complex systems, namely, those depending on an arbitrary number $n$ of random Boolean variables. That is, the $n$ basic variables $x_{1}, \ldots, x_{n}$ of the system are assumed to be stochastic and they only take two possible values, 0 or 1 , with probabilities

$$
\operatorname{Pr}\left\{x_{i}=1\right\}=p_{i}, \quad \operatorname{Pr}\left\{x_{i}=0\right\}=1-p_{i},
$$

where the values $\left\{p_{i}\right\}_{i=1}^{n}$ will be referred to as the basic probabilities or parameters of the system.

We call such a system a complex stochastic Boolean system (CSBS). These systems can be found in many different scientific or engineering areas like mechanical engineering, meteorology and climatology, nuclear physics, complex systems analysis, operations research, and so forth. CSBSs also arise very often when analyzing system safety in reliability engineering and risk analysis; see, for example, [1-3].

Each one of the $2^{n}$ possible outcomes associated with a CSBS is given by a binary $n$-tuple (or bitstring of length $n$ ) $u=\left(u_{1}, \ldots, u_{n}\right) \in\{0,1\}^{n}$, and it has its own occurrence probability $\operatorname{Pr}\{u\}$.
Throughout this paper, the $n$ Boolean variables $x_{i}$ of the CSBS are assumed to be mutually independent, so that the occurrence probability of a given binary string $u$ of length $n$ can be easily computed as

$$
\operatorname{Pr}\{u\}=\prod_{i=1}^{n} p_{i}^{u_{i}}\left(1-p_{i}\right)^{1-u_{i}} \quad \forall u \in\{0,1\}^{n}
$$

that is, $\operatorname{Pr}\{u\}$ is the product of factors $p_{i}$ if $u_{i}=1,1-p_{i}$ if $u_{i}=0$.

As an example of CSBS, we can consider a technical system like the accumulator system of a pressured water reactor in a nuclear power plant, taken from [4]. This technical system depends on $n=83$ mutually independent basic components $x_{1}, \ldots, x_{83}$. Assuming that $x_{i}=1$ if component $i$ fails, $x_{i}=0$ otherwise; then the failure and working probabilities of component $i$ will be $\operatorname{Pr}\left\{x_{i}=1\right\}=p_{i}, \operatorname{Pr}\left\{x_{i}=\right.$ $0\}=1-p_{i}$, respectively. The probability $p_{i}$ of failure of each basic component $i(1 \leq i \leq 83)$ is given, so that all basic probabilities $p_{i}$ are known.

Thus, this accumulator system can be considered as a CSBS where each one of its $2^{83}$ system binary states (i.e., binary 83-tuple $\left.\left(u_{1}, \ldots, u_{83}\right) \in\{0,1\}^{83}\right)$ describes the current 
situation of its 83 basic components (failing or working). For instance, the binary 83-tuple

$$
u=(1, \ldots, \ldots, 1,0, \ldots, 0)
$$

represents the system state for which the first 23 components fail, while the last 60 components work. Moreover, the occurrence probability of the binary state $u$ can be immediately computed using (2) as follows:

$$
\operatorname{Pr}\{u\}=\prod_{i=1}^{23} p_{i} \prod_{i=24}^{83}\left(1-p_{i}\right) .
$$

Real-world CSBSs arising in many different engineering areas, like the above-mentioned accumulator system, are typically analyzed in many works dealing with system safety and reliability theory. In this context, let us mention that formula (2) for computing the binary $n$-tuple probabilities associated with a CSBS on $n$-independent Boolean variables (basic components) can be seen as a particular case of Theorem 1 in [5]. In that theorem, the author considers a coherent or noncoherent $n$ component system with dependent or independent failures and proves that the reliability/unreliability function $G(x)$ in the system is a multilinear function of the vector $x$ of all conditional component (success/failure) probabilities. Let us recall that a system is said to be coherent if its structure (Boolean) function $\phi$ satisfies [5]

$$
\phi(0, \ldots, 0)=0, \quad \phi(1, \ldots, 1)=1,
$$

and if $u, v \in\{0,1\}^{n}$ are such that $u_{i} \leq v_{i}$ for all $i=1,2, \ldots, n$, and $u_{i}<v_{i}$ for some $i$, then

$$
\phi\left(u_{1}, \ldots, u_{n}\right)<\phi\left(v_{1}, \ldots, v_{n}\right) .
$$

Moreover, we must highlight that the assumption of independent failures (an essential hypothesis for formula (2) and for the results presented in our paper), while being classical and advantageous from a calculation point of view, is a bit restrictive for realistic applications. In this respect, in [6] one can find an illustration of the issues when realistic systems are addressed.

The behavior of a CSBS is determined by the ordering between the current values of the $2^{n}$ associated binary $n$-tuple probabilities $\operatorname{Pr}\{u\}$. Due to the exponential complexity of the problem, computing all these $2^{n}$ probabilities - by using (2) and ordering them in decreasing or increasing order of their values is only possible in practice for small values of the number $n$ of basic variables. However, for large values of $n$, it is necessary to use alternative procedures to compare the binary string probabilities. For this purpose, a simple, positional criterion to order binary $n$-tuple probabilities is used. The socalled intrinsic order criterion (IOC) enables one to compare (to order) the occurrence probabilities $\operatorname{Pr}\{u\}, \operatorname{Pr}\{v\}$ of two given binary $n$-tuples $u, v$ without the need to compute them, simply looking at the relative positions of their 0 s and $1 \mathrm{~s}$. IOC was first described in [7], and it naturally leads to a partial order relation on the set $\{0,1\}^{n}$ of all the binary strings of length $n$. The so-called intrinsic order provides a unified approach for the analysis and modeling of CSBSs.
The most useful representation of a CSBS is the intrinsic order graph: a symmetric directed graph on $2^{n}$ vertices, displaying all the $2^{n}$ binary $n$-tuples from top to bottom in decreasing order of their occurrence probabilities.

In this context, the main goal of this paper is to compare the occurrence probabilities of two binary strings with the same length $n$ and containing the same number of 1-bits. Our results will be derived from IOC, as well as from other properties of the intrinsic ordering, and they will be illustrated with the intrinsic order graph.

For this purpose, this paper has been organized as follows. Section 2 contains all the background about the intrinsic order relation, required to make this paper self-contained. Section 3 is devoted to present our new results concerning the comparison between the occurrence probabilities of two binary strings with the same number of 1-bits. Finally, conclusions are presented in Section 4.

\section{Preliminaries}

Let us start this section with some basic concepts and notations which will be used in the rest of the paper.

Definition 1. Let $n \geq 1$ and let $u=\left(u_{1}, \ldots, u_{n}\right) \in\{0,1\}^{n}$ be a binary $n$-tuple. Then we have the following.

(i) The decimal equivalent of $u$ will be denoted by $u_{(10}$ (to avoid confusion with the 10th component $u_{10}$ of the binary vector $u$ ); that is,

$$
u_{(10}=\sum_{i=1}^{n} 2^{n-i} u_{i}
$$

In what follows, we indistinctly denote the $n$-tuple $u \in\{0,1\}^{n}$ by its binary representation $\left(u_{1}, \ldots, u_{n}\right)$ or by its decimal representation $u_{(10}$, and we use the symbol " $\equiv$ " to denote the conversion between both representations; that is,

$$
\left(u_{1}, \ldots, u_{n}\right) \equiv u_{(10} .
$$

(ii) The Hamming weight (or simply the weight) $w_{H}(u)$ of $u$ is the number of 1 -bits in $u$; that is,

$$
w_{H}(u)=\sum_{i=1}^{n} u_{i}
$$

(iii) The complementary $n$-tuple of $u$ is the $n$-tuple obtained by changing all its 0 s to 1 s and vice versa; that is,

$$
\left(u_{1}, \ldots, u_{n}\right)^{c}=\left(1-u_{1}, \ldots, 1-u_{n}\right) .
$$

Note that two binary $n$-tuples are complementary if and only if they sum up to $(1, \ldots, 1) \equiv 2^{n}-1$. Then, using the decimal numbering, the complementary $n$-tuple $u^{c}$ of a binary $n$-tuple $u$ is given by

$$
u_{(10}^{c}=\left(2^{n}-1\right)-u_{(10} .
$$


Example 2. Let $n=5$ and $u=(0,1,1,0,1) \in\{0,1\}^{5}$. Then

(i) $(0,1,1,0,1) \equiv 2^{0}+2^{2}+2^{3}=13=u_{(10}$;

(ii) $w_{H}(0,1,1,0,1)=3$;

(iii) $13^{c} \equiv(0,1,1,0,1)^{c}=(1,0,0,1,0) \equiv 18$;

$$
13+13^{c}=13+18=31=2^{5}-1 .
$$

2.1. The Intrinsic Order Criterion. According to (2), the ordering between two given binary string probabilities $\operatorname{Pr}(u)$ and $\operatorname{Pr}(v)$ depends, in general, on the parameters $p_{i}$, as the following simple example shows.

Example 3. Let $n=3, u=(0,1,1)$, and $v=(1,0,0)$. Using (2), we have

$$
\begin{aligned}
& p_{1}=0.1, \quad p_{2}=0.2, \quad p_{3}=0.3: \\
& \operatorname{Pr}\{u\}=0.054<\operatorname{Pr}\{v\}=0.056, \\
& p_{1}=0.2, \quad p_{2}=0.3, \quad p_{3}=0.4: \\
& \operatorname{Pr}\{u\}=0.096>\operatorname{Pr}\{v\}=0.084 .
\end{aligned}
$$

However, assuming a simple (but not restrictive in practice) hypothesis on the parameters $p_{i}$, we can assure that for some pairs of binary $n$-tuples, the ordering between their occurrence probabilities is independent of the basic probabilities $p_{i}$. More precisely, as mentioned in Section 1, to overcome the exponential complexity in the problem of computing and sorting the $2^{n}$ binary string probabilities, the following simple positional criterion has been introduced in [7].

Theorem 4 (the intrinsic order theorem). Let $n \geq 1$. Let $x_{1}, \ldots, x_{n}$ be $n$ mutually independent stochastic Boolean variables whose parameters $p_{i}=\operatorname{Pr}\left\{x_{i}=1\right\}$ satisfy

$$
0<p_{1} \leq p_{2} \leq \cdots \leq p_{n} \leq \frac{1}{2}
$$

Then the probability of the binary $n$-tuple $v=\left(v_{1}, \ldots, v_{n}\right)$ is (intrinsically) less than or equal to the probability of the binary $n$-tuple $u=\left(u_{1}, \ldots, u_{n}\right)$ (here, "intrinsically" means: for all set $\left\{p_{i}\right\}_{i=1}^{n}$ satisfying (13)) if and only if either the matrix

$$
M_{v}^{u}:=\left(\begin{array}{lll}
u_{1} & \cdots & u_{n} \\
v_{1} & \cdots & v_{n}
\end{array}\right)
$$

has no $\left(\begin{array}{l}1 \\ 0\end{array}\right)$ columns or for each $\left(\begin{array}{l}1 \\ 0\end{array}\right)$ column in $M_{v}^{u}$ there exists (at least) one corresponding preceding $\left(\begin{array}{l}0 \\ 1\end{array}\right)$ column (IOC).

Remark 5. In the following, we assume that the parameters $p_{i}$ always satisfy condition (13). Note that this hypothesis is not restrictive for practical applications because for any index $i$ such that $p_{i}>1 / 2$, we only need to consider the variable $\overline{x_{i}}=1-x_{i}$, instead of $x_{i}$. Next, we order the $n$ new Boolean variables in increasing order of their probabilities. This reduces the complexity in sorting tasks from exponential (ordering $2^{n}$ binary $n$-tuple probabilities) to linear (ordering $n$ Boolean variable probabilities).
Remark 6. The $\left(\begin{array}{l}0 \\ 1\end{array}\right)$ column preceding each $\left(\begin{array}{l}1 \\ 0\end{array}\right)$ column is not required to be necessarily placed at the immediately previous position, but just at previous position.

Remark 7. The term "corresponding," used in Theorem 4, has the following meaning: for each two $\left(\begin{array}{l}1 \\ 0\end{array}\right)$ columns in matrix $M_{v}^{u}$ there must exist (at least) two different $\left(\begin{array}{l}0 \\ 1\end{array}\right)$ columns preceding each other. Formally, there must exist (at least) one injective precedence map from the set of $\left(\begin{array}{l}1 \\ 0\end{array}\right)$ columns of $M_{v}^{u}$ onto the set of its $\left(\begin{array}{l}0 \\ 1\end{array}\right)$ columns that assigns to each $\left(\begin{array}{l}1 \\ 0\end{array}\right)$ column a $\left(\begin{array}{l}0 \\ 1\end{array}\right)$ column preceding it. In other words, for each $\left(\begin{array}{l}1 \\ 0\end{array}\right)$ column in matrix $M_{v}^{u}$, the number of preceding $\left(\begin{array}{l}0 \\ 1\end{array}\right)$ columns must be strictly greater than the number of preceding $\left(\begin{array}{l}1 \\ 0\end{array}\right)$ columns.

The matrix condition IOC, stated by Theorem 4, is called the intrinsic order criterion because it is independent of the basic probabilities $p_{i}$, and it only depends on the relative positions of the bits in the binary $n$-tuples $u$ and $v$ to be compared. Theorem 4 naturally leads to the following partial order relation on the set $\{0,1\}^{n}$; see [7]. The so-called intrinsic order will be denoted by “ $\preceq$ ", and when $v \preceq u$ we will say that $v$ is intrinsically less than or equal to $u$.

Definition 8. For all $u, v \in\{0,1\}^{n}$,

$$
\begin{gathered}
v \preceq u \quad \text { iff } \operatorname{Pr}\{v\} \leq \operatorname{Pr}\{u\} \quad \text { for all set }\left\{p_{i}\right\}_{i=1}^{n} \\
\text { s.t. (13) iff } M_{v}^{u} \text { satisfies IOC. }
\end{gathered}
$$

From now on, the partially ordered set (poset, for short) $\left(\{0,1\}^{n}, \preceq\right)$ will be denoted by $I_{n}$.

Example 9. For $n=3$, we have $3 \equiv(0,1,1) \npreceq 4 \equiv(1,0,0)$ and $4 \equiv(1,0,0) \npreceq 3 \equiv(0,1,1)$ because the matrices

$$
M_{3}^{4}=\left(\begin{array}{lll}
1 & 0 & 0 \\
0 & 1 & 1
\end{array}\right), \quad M_{4}^{3}=\left(\begin{array}{lll}
0 & 1 & 1 \\
1 & 0 & 0
\end{array}\right)
$$

do not satisfy IOC (Remark 7$)$. Thus, $(0,1,1)$ and $(1,0,0)$ are incomparable by intrinsic order; that is, the ordering between the two probabilities $\operatorname{Pr}\{(0,1,1)\}$ and $\operatorname{Pr}\{(1,0,0)\}$ depends on the current values of parameters $\left\{p_{i}\right\}_{i=1}^{3}$, as Example 3 has shown.

Example 10. For $n=4$, we have $3 \equiv(0,0,1,1)>12 \equiv$ $(1,1,0,0)$ because matrix

$$
M_{12}^{3}=\left(\begin{array}{llll}
0 & 0 & 1 & 1 \\
1 & 1 & 0 & 0
\end{array}\right)
$$

satisfies IOC (Remark 6). Thus, for all $\left\{p_{i}\right\}_{i=1}^{4}$ s.t. (13),

$$
\operatorname{Pr}\{(1,1,0,0)\} \leq \operatorname{Pr}\{(0,0,1,1)\} .
$$

Example 11. For $n=1007$, we have

$$
86 \equiv(0, . . ., 0,1,0,1,0,1,1,0) \preceq 35 \equiv(0, . . ., 0,0,1,0,0,0,1,1)
$$


because matrix

$$
M_{86}^{35}=\left(\begin{array}{cccccccccc}
0 & \stackrel{1000}{\cdots} & 0 & 0 & 1 & 0 & 0 & 0 & 1 & 1 \\
0 & \stackrel{1000}{10} & 0 & 1 & 0 & 1 & 0 & 1 & 1 & 0
\end{array}\right)
$$

satisfies IOC (Remark 6). Thus, for all $\left\{p_{i}\right\}_{i=1}^{1007}$ s.t. (13),

$$
\begin{aligned}
& \operatorname{Pr}\{(0, \stackrel{1000}{.}, 0,1,0,1,0,1,1,0)\} \\
& \leq \operatorname{Pr}\{(0, \stackrel{1000}{.} 0,0,1,0,0,0,1,1)\} .
\end{aligned}
$$

Example 12. For all $n \geq 1$, the binary $n$-tuples

$$
(0, \ldots, \stackrel{n}{.}, 0) \equiv 0, \quad(1, \ldots, \ldots, 1) \equiv 2^{n}-1
$$

are the maximum and minimum elements, respectively, in the poset $I_{n}$. Indeed, for every binary $n$-tuple $\left(u_{1}, \ldots, u_{n}\right) \in$ $\{0,1\}^{n}$, both matrices

$$
\left(\begin{array}{ccc}
0 & \cdots & 0 \\
u_{1} & \cdots & u_{n}
\end{array}\right), \quad\left(\begin{array}{ccc}
u_{1} & \cdots & u_{n} \\
1 & \cdots & 1
\end{array}\right)
$$

satisfy IOC, since they have no $\left(\begin{array}{l}1 \\ 0\end{array}\right)$ columns! Thus, for all binary $n$-tuples $\left(u_{1}, \ldots, u_{n}\right) \in\{0,1\}^{n}$, and for all $\left\{p_{i}\right\}_{i=1}^{n}$ s.t. (13),

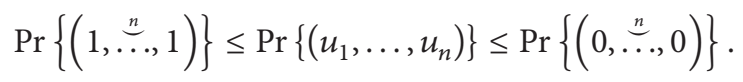

Many different properties of the intrinsic order relation can be derived from its simple matrix description IOC. For the purpose of this paper, it suffices to recall here the following necessary (but not sufficient) condition for intrinsic order.

Note that if $u \geq v$, then there must exist (at least) one injective precedence map from the set of $\left(\begin{array}{l}1 \\ 0\end{array}\right)$ columns of $M_{v}^{u}$ onto the set of its $\left(\begin{array}{l}0 \\ 1\end{array}\right)$ columns (Remark 7). Then in matrix $M_{v}^{u}$ the number of $\left(\begin{array}{l}1 \\ 0\end{array}\right)$ columns must be less than or equal to the number of $\left(\begin{array}{l}0 \\ 1\end{array}\right)$ columns. But this is equivalent to saying that the number of 1-bits in $u$ must be less than or equal to the number of 1-bits in $v$. That is

Corollary 13. The intrinsic order respects the Hamming weight. More precisely, for all, $v \in\{0,1\}^{n}$,

$$
u \geq v \Longrightarrow w_{H}(u) \leq w_{H}(v) .
$$

The converse of Corollary 13 is false, as the following two simple counterexamples (indeed, the simplest ones that one can find) show.

(i) For $n=2, u=2 \equiv(1,0)$, and $v=1 \equiv(0,1)$, we have

$$
w_{H}(u)=1=w_{H}(v), \quad u \nsucceq v
$$

since matrix

$$
M_{1}^{2}=\left(\begin{array}{ll}
1 & 0 \\
0 & 1
\end{array}\right)
$$

does not satisfy IOC.

(ii) For $n=3$ and for $u=4 \equiv(1,0,0), v=3 \equiv(0,1,1)$, we have (see Example 9)

$$
w_{H}(u)=1<2=w_{H}(v), \quad u \nsucceq v .
$$

\author{
1 \\ FIgURE 1: The intrinsic order graph for $n=1$.
}

2.2. The Intrinsic Order Graph. To finish this section, we present the graphical representation of the poset $I_{n}$. As it is well known, the usual representation for a poset is its Hasse diagram (see [8] for more details about posets and their diagrams). Let us recall that, for a poset $(P, \leq)$ and for $x, y \in P$, we say that $y$ covers $x$ if $x<y$ with no other elements between them. The Hasse diagram of a finite poset $(P, \leq)$ is the graph whose vertices are the elements of $P$ and whose edges are the cover relations (with the convention that if $y$ covers $x$, then $y$ is drawn above $x$ ). The Hasse diagram of our poset $I_{n}$ will be also called the intrinsic order graph for $n$ variables, denoted as well by $I_{n}$. So, this is a directed graph (digraph, for short) whose vertices are the $2^{n}$ binary $n$ tuples of 0 s and $1 \mathrm{~s}$, and whose edges go downward from $u$ to $v$ whenever $u$ covers $v$ (denoted by $u \triangleright v$ ); that is,

$$
\begin{array}{r}
u \triangleright v \text { iff } u>v \text { and there is no } w \in\{0,1\}^{n} \\
\text { s.t. } u>w>v .
\end{array}
$$

For small values of $n$, the intrinsic order graph $I_{n}$ can be directly constructed by using Theorem 4 . For instance, for $n=$ 1 , the Hasse diagram of $I_{1}=(\{0,1\}, \preceq)$ is shown in Figure 1 . Indeed, using Theorem 4 , we have that $0>1$, since matrix $\left(\begin{array}{l}1 \\ 0\end{array}\right)$ has no $\left(\begin{array}{l}1 \\ 0\end{array}\right)$ columns! So, $I_{1}$ has a downward edge from 0 to 1 , and this is in accordance with the fact that

$$
\operatorname{Pr}\{0\}=1-p_{1} \geq p_{1}=\operatorname{Pr}\{1\},
$$

since $p_{1} \leq 1 / 2$ due to (13).

However, for large values of $n$, a more efficient method is needed. For this purpose, in [9] the following algorithm for iteratively building up $I_{n}$ (for all $n \geq 2$ ) from $I_{1}$ (depicted in Figure 1) has been developed.

Theorem 14 (building up $I_{n}$ from $\left.I_{1}\right)$. Let $n \geq 2$. The graph of the poset $I_{n}=\left\{0, \ldots, 2^{n}-1\right\}$ (on $2^{n}$ nodes) can be drawn simply by adding to the graph of the poset $I_{n-1}=\left\{0, \ldots, 2^{n-1}-1\right\}$ (on $2^{n-1}$ nodes) its isomorphic copy $2^{n-1}+I_{n-1}=\left\{2^{n-1}, \ldots, 2^{n}-1\right\}$ (on $2^{n-1}$ nodes). This addition must be performed by placing the powers of 2 at consecutive levels in the Hasse diagram of $I_{n}$. Finally, the edges connecting one vertex $u$ of $I_{n-1}$ with the other vertex $v$ of $2^{n-1}+I_{n-1}$ are given by the set of vertex pairs

$$
\left\{(u, v)=\left(u_{(10}, 2^{n-2}+u_{(10}\right) \mid 2^{n-2} \leq u_{(10} \leq 2^{n-1}-1\right\} .
$$

In Figure 2, the iterative process described in Theorem 14 is illustrated by showing the intrinsic order graphs for $n=$ $1,2,3,4$ from left to right. Basically, to draw $I_{n}$, we first add to $I_{n-1}$ its isomorphic copy $2^{n-1}+I_{n-1}$, and then we connect one-to-one the nodes of "the second half of the first half" to the nodes of "the first half of the second half." Hence, the intrinsic order graph $I_{n}$ is a fractal or self-similar graph 


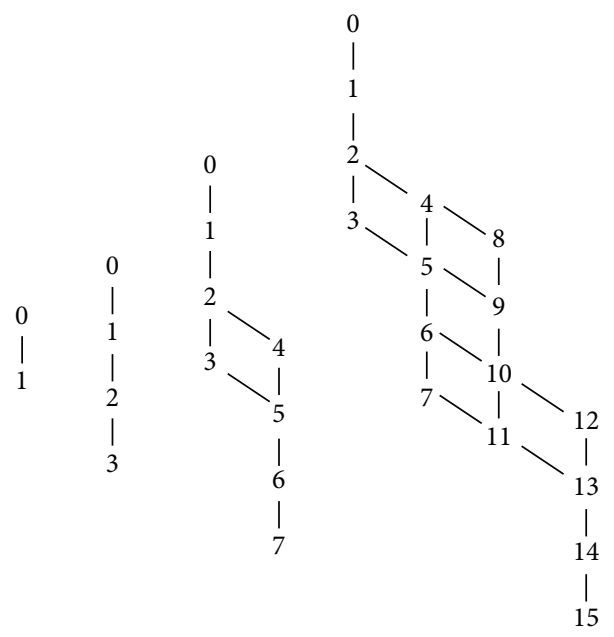

FiguRE 2: The intrinsic order graphs for $n=1,2,3,4$ using decimal representation.

in the sense that it can be recursively constructed from the previous $\left(I_{1}, I_{2}, \ldots, I_{n-1}\right)$ by some operations which preserve self-similarity; that is, it appears similar at different scales (orders). In Figure 3, the intrinsic order graphs for $n=3,4$ are depicted using the binary representation instead of the decimal representation of their nodes.

Note that $I_{n}$ has a downward path from $u$ to $v$ if and only if $u>v$. For instance, looking at the digraph $I_{4}$ (the rightmost one in Figure 2), we confirm that $3>12$, as shown in Example 10.

On the contrary, each pair $(u, v)$ of nonconnected vertices in the digraph of $I_{n}$, either by one edge or by a longer downward path, means that $u$ and $v$ are incomparable by intrinsic order; that is, $u \nsucceq v$ and $v \nsucceq u$. For instance, looking at the digraph $I_{3}$ (the third one from the left in Figure 2), we confirm that $3 \nsucceq 4$ and $4 \nsucceq 3$, as shown in Example 3 or in Example 9.

Also, looking at any of the digraphs in Figure 2, we can confirm Corollary 13 , as well as the fact that 0 and $2^{n}-1$ are the maximum and minimum elements, respectively, in the poset $I_{n}$, as shown in Example 12.

Recall that two binary $n$-tuples are complementary if and only if their decimal equivalents sum up to $2^{n}-1$ (see Definition 1-(iii)). Hence, one can observe that any two complementary $n$-tuples are placed at symmetric positions (with respect to the central point) in the intrinsic order graph $I_{n}$. For instance, this is the case in the graph $I_{4}$ for the following pairs of binary 4-tuples (see the right-most graphs in Figures 2 and 3):

$$
\begin{gathered}
u=(0,0,1,1) \equiv 3, \quad u^{c}=(1,1,0,0) \equiv 12, \\
u+u^{c}=3+12=15=2^{4}-1, \\
u=(0,1,1,0) \equiv 6, \quad u^{c}=(1,0,0,1) \equiv 9, \\
u+u^{c}=6+9=15=2^{4}-1, \\
u=(0,1,1,1) \equiv 7, \quad u^{c}=(1,0,0,0) \equiv 8, \\
u+u^{c}=7+8=15=2^{4}-1 .
\end{gathered}
$$

The edgeless graph associated with a given graph is obtained by removing all its edges, keeping its (isolated) nodes at the same positions. In Figures 4 and 5, the edgeless intrinsic order graphs for $I_{5}$ and $I_{6}$, respectively, are depicted.

For further theoretical properties and practical applications of the intrinsic order and the intrinsic order graph, we refer the reader to $[7,9-13]$ and to the references therein.

\section{Occurrence Probabilities of Bitstrings with the Same Weight}

In this section, we present our results about the comparison, by intrinsic order, between the occurrence probabilities of two binary $n$-tuples having the same Hamming weight.

For the special case that the bitstrings $u, v \in\{0,1\}^{n}$ have the same weight, the intrinsic order can be characterized as stated by the following two lemmas.

Lemma 15. Let $n \geq 1$. Let $u, v \in\{0,1\}^{n}$ with $w_{H}(u)=w_{H}(v)$. Then $u \geq v$ if and only if the matrix

$$
M_{v}^{u}=\left(\begin{array}{lll}
u_{1} & \cdots & u_{n} \\
v_{1} & \cdots & v_{n}
\end{array}\right)
$$

has neither $\left(\begin{array}{l}1 \\ 0\end{array}\right)$ nor $\left(\begin{array}{l}0 \\ 1\end{array}\right)$ columns, or for each $\left(\begin{array}{l}1 \\ 0\end{array}\right)$ column in $M_{v}^{u}$ there exists exactly one corresponding preceding $\left(\begin{array}{l}0 \\ 1\end{array}\right)$ column.

Proof. Using Definition 8 and Theorem 4, we have that $u \geq v$ if and only if matrix $M_{v}^{u}$ satisfies IOC, if and only if either matrix $M_{v}^{u}$ has no $\left(\begin{array}{l}1 \\ 0\end{array}\right)$ columns or for each $\left(\begin{array}{l}1 \\ 0\end{array}\right)$ column in matrix $M_{v}^{u}$ there exists at least one corresponding preceding $\left(\begin{array}{l}0 \\ 1\end{array}\right)$ column (IOC). But, under the assumption that $u$ and $v$ have the same number of 1-bits, IOC is actually equivalent to saying that matrix $M_{v}^{u}$ has neither $\left(\begin{array}{l}1 \\ 0\end{array}\right)$ nor $\left(\begin{array}{l}0 \\ 1\end{array}\right)$ columns (in this case, obviously, $u=v$ ), or for each $\left(\begin{array}{l}1 \\ 0\end{array}\right)$ column in matrix $M_{v}^{u}$ there exists exactly one corresponding preceding $\left(\begin{array}{l}0 \\ 1\end{array}\right)$ column. 

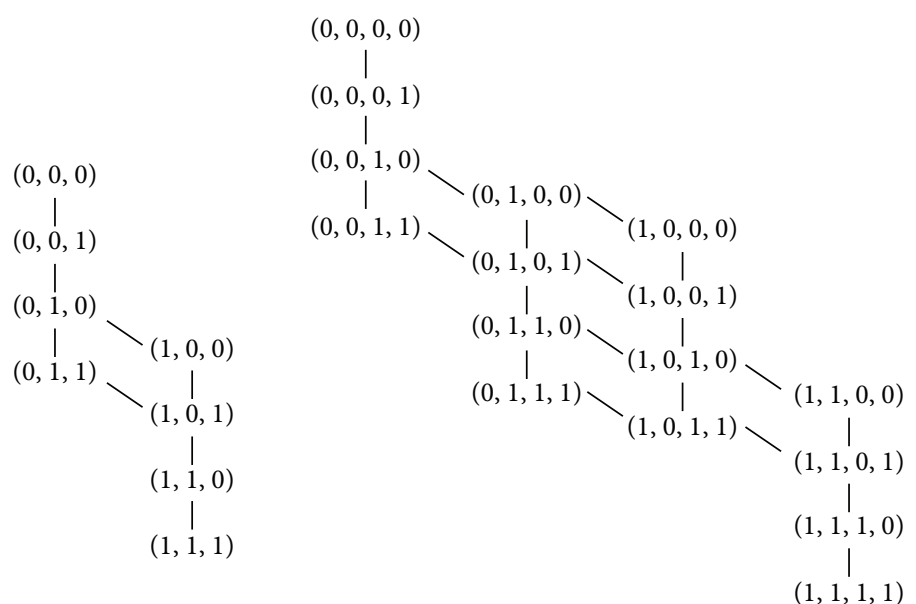

FIGURE 3: The intrinsic order graphs for $n=3,4$ using binary representation.

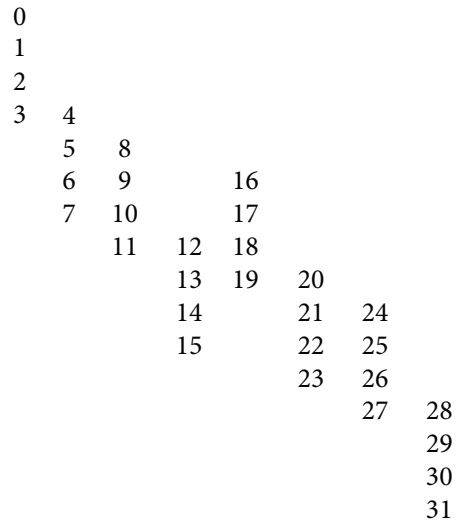

Figure 4: The edgeless intrinsic order graph for $n=5$.

The following lemma characterizes the intrinsic order between binary $n$-tuples of the same weight, involving only their 1-bits, with no need to use their 0-bits.

Lemma 16. Let $n \geq 1$. Let $u, v \in\{0,1\}^{n}$ with $w_{H}(u)=w_{H}(v)$. Then $u \geq v$ if and only if for each 1-bit in $u$ there exists exactly one corresponding 1-bit in $v$ placed at the same or at a previous position.

Proof. Using Lemma 15, we have that $u \geq v$ if and only if matrix $M_{v}^{u}$ has neither $\left(\begin{array}{l}1 \\ 0\end{array}\right)$ nor $\left(\begin{array}{l}0 \\ 1\end{array}\right)$ columns, or each $\left(\begin{array}{l}1 \\ 0\end{array}\right)$ column in $M_{v}^{u}$ is preceded by exactly one corresponding $\left(\begin{array}{l}0 \\ 1\end{array}\right)$ column. On one hand, each $\left(\begin{array}{l}1 \\ 1\end{array}\right)$ column in $M_{v}^{u}\left(\operatorname{say}\left(\begin{array}{c}\mathcal{u}_{i} \\ v_{i}\end{array}\right)=\right.$ $\left.\left(\begin{array}{l}1 \\ 1\end{array}\right)\right)$ corresponds to a 1-bit placed at the same position in both binary $n$-tuples $u$ and $v\left(u_{i}=v_{i}=1\right)$. On the other hand, each column $\left(\begin{array}{l}u_{i} \\ v_{i}\end{array}\right)=\left(\begin{array}{l}1 \\ 0\end{array}\right)$ in $M_{v}^{u}$ preceded by its corresponding column $\left(\begin{array}{c}u_{j} \\ v_{j}\end{array}\right)=\left(\begin{array}{c}0 \\ 1\end{array}\right)(j<i)$ corresponds to a 1-bit $v_{j}=1$ in $v$ placed at a previous position than the 1-bit $u_{i}=1$ in $u$.

Now, we introduce the following notation for binary $n$ tuples.
Definition 17. Let $n \geq 1$ and let $u \in\{0,1\}^{n}$ with Hamming weight $w_{H}(u)=m$. Then

(i) the vector of positions of $1 \mathrm{~s}$ of $u$ is the vector of positions of its $m 1$-bits, displayed in increasing order from the left-most position to the right-most position, and it will be denoted by

$$
\begin{gathered}
u=\left[i_{1}, i_{2}, \ldots, i_{m}\right]_{n}^{1}, \\
1 \leq i_{1}<i_{2}<\cdots<i_{m} \leq n, \quad 0<m \leq n ;
\end{gathered}
$$

(ii) the vector of positions of 0 s of $u$ is the vector of positions of its $(n-m) 0$-bits, displayed in increasing order from the left-most position to the right-most position, and it will be denoted by

$$
\begin{gathered}
u=\left[\bar{i}_{1}, \bar{i}_{2}, \ldots, \bar{i}_{n-m}\right]_{n}^{0}, \\
1 \leq \bar{i}_{1}<\bar{i}_{2}<\cdots<\bar{i}_{n-m} \leq n, \quad 0 \leq m<n .
\end{gathered}
$$

We also use the symbol " $\equiv$ " to denote the conversion between any of these vector notations and the binary or decimal representation of the bitstrings.

Example 18. Let $n=7$ and $u=43 \equiv(0,1,0,1,0,1,1)$. Then we have $m=w_{H}(u)=4, n-m=3$, and

$$
\begin{gathered}
u=(0,1,0,1,0,1,1) \equiv\left[i_{1}, i_{2}, i_{3}, i_{4}\right]_{7}^{1}=[2,4,6,7]_{7}^{1}, \\
u=(0,1,0,1,0,1,1) \equiv\left[\bar{i}_{1}, \bar{i}_{2}, \bar{i}_{3}\right]_{7}^{0}=[1,3,5]_{7}^{0} .
\end{gathered}
$$

The following theorem characterizes the intrinsic order between binary $n$-tuples of the same weight, using the vectors of positions of their 1-bits, introduced in Definition 17-(i).

Theorem 19. Let $n \geq 1$. Let $u, v \in\{0,1\}^{n}$ with $w_{H}(u)=$ $w_{H}(v)$. Let

$$
\begin{gathered}
u=\left[i_{1}, i_{2}, \ldots, i_{m}\right]_{n}^{1}, \quad v=\left[j_{1}, j_{2}, \ldots, j_{m}\right]_{n}^{1}, \\
\left(\begin{array}{c}
1 \leq i_{1}<i_{2}<\cdots<i_{m} \leq n \\
1 \leq j_{1}<j_{2}<\cdots<j_{m} \leq n
\end{array}\right)
\end{gathered}
$$




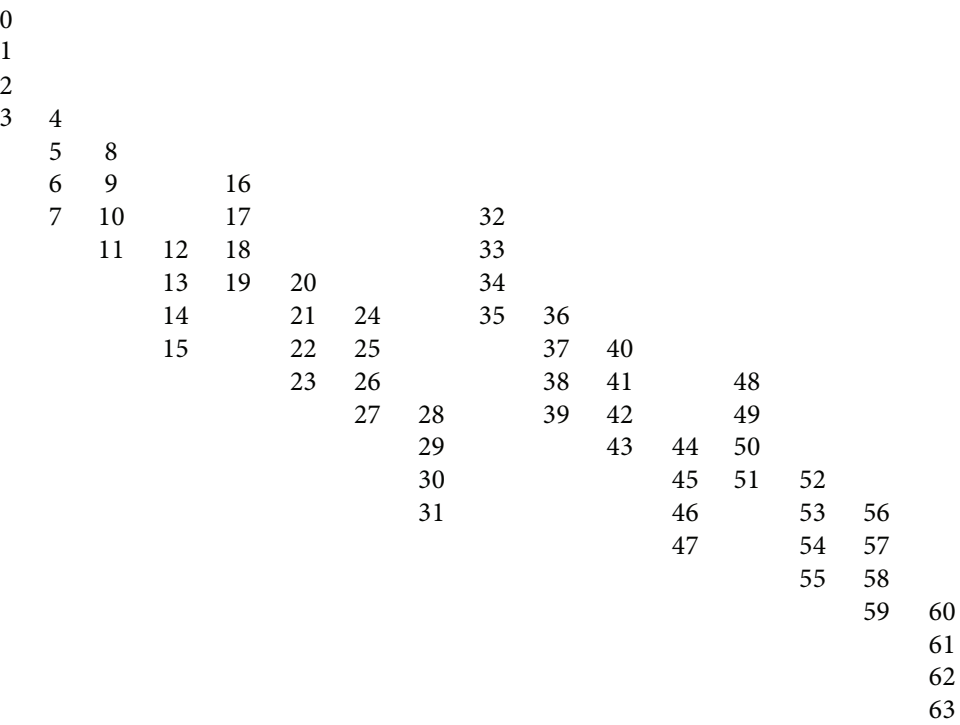

FIGURE 5: The edgeless intrinsic order graph for $n=6$.

be the vectors of positions of 1-bits of $u$ and $v$, respectively. Then

$$
u \geq v \Longleftrightarrow v \preceq u \Longleftrightarrow j_{1} \leq i_{1}, \quad j_{2} \leq i_{2}, \ldots, j_{m} \leq i_{m} .
$$

Proof. Using Lemma 16, we have that $u \geq v$ if and only if for each 1-bit in $u$ there exists exactly one corresponding 1-bit in $v$ placed at the same or at a previous position. Now, according to Definition 17-(i), sweeping the $m 1$-bits of $u$ from left to right, the last assertion is clearly equivalent to saying that

$$
j_{1} \leq i_{1}, j_{2} \leq i_{2}, \ldots, j_{m} \leq i_{m}
$$

and the proof is concluded.

Example 20. Let $n=9$ and let

$$
\begin{aligned}
u & =(0,0,0,1,1,0,1,1,1) \equiv\left[i_{1}, i_{2}, i_{3}, i_{4}, i_{5}\right]_{9}^{1} \\
& =[4,5,7,8,9]_{9}^{1}, \\
v & =(1,1,0,0,1,1,0,0,1) \equiv\left[j_{1}, j_{2}, j_{3}, j_{4}, j_{5}\right]_{9}^{1} \\
& =[1,2,5,6,9]_{9}^{1} .
\end{aligned}
$$

Using Theorem 4 , we have that $v \preceq u$ since matrix

$$
M_{v}^{u}=\left(\begin{array}{lllllllll}
0 & 0 & 0 & 1 & 1 & 0 & 1 & 1 & 1 \\
1 & 1 & 0 & 0 & 1 & 1 & 0 & 0 & 1
\end{array}\right) \quad \text { satisfies IOC. }
$$

Now, since $w_{H}(u)=w_{H}(v)=5$, then alternatively, we can apply Theorem 19 . In this way, we also conclude that $v \preceq u$, since

$$
j_{1}<i_{1}, j_{2}<i_{2}, j_{3}<i_{3}, j_{4}<i_{4}, j_{5}=i_{5}
$$

Given a fixed binary $n$-tuple $u \in\{0,1\}^{n}$ with weight $m$, the following theorem provides us with the set and with the number of all the binary $n$-tuples $v \in\{0,1\}^{n}$ with weight $m$ that are intrinsically less than or equal to $u$ (i.e., $v \preceq u$ ). That is, it provides the set and the number of all the binary $n$-tuples $v$ with the same weight as $u$, and whose occurrence probabilities are always (i.e., intrinsically) less than or equal to the occurrence probability of $u$.

Theorem 21. Let $u \in\{0,1\}^{n}, u \neq 0$, with (nonzero) Hamming weight $w_{H}(u)=m(0<m \leq n)$. Let $u=\left[i_{1}, i_{2}, \ldots, i_{m}\right]_{n}^{1}$ be the vector of positions of $1 s$ of $u$. Then the set of the binary $n$-tuples $v$ with weight $m$, which are intrinsically less than or equal to $u$, is given by

$$
\begin{gathered}
\left\{v \in\{0,1\}^{n} \mid w_{H}(v)=w_{H}(u), v \preceq u\right\} \\
=\left\{v=\left[j_{1}, j_{2}, \ldots, j_{m}\right]_{n}^{1} \mid j_{1} \leq i_{1},\right. \\
\left.j_{2} \leq i_{2}, \ldots, j_{m} \leq i_{m}\right\} .
\end{gathered}
$$

Moreover, the number of the binary $n$-tuples $v$ with weight $m$, that are intrinsically less than or equal to $u$, is given by

$$
\begin{gathered}
\left|\left\{v \in\{0,1\}^{n} \mid w_{H}(v)=w_{H}(u), v \preceq u\right\}\right| \\
=\sum_{j_{1}=1}^{i_{1}} \sum_{j_{2}=j_{1}+1}^{i_{2}} \ldots \sum_{j_{m}=j_{m-1}+1}^{i_{m}} 1 .
\end{gathered}
$$

Proof. Using Theorem 19, we have that

$$
v \preceq u \Longleftrightarrow j_{p} \leq i_{p} \quad \forall p=1,2, \ldots, m
$$

and this proves (45). Finally, (46) immediately follows from the fact that $1 \leq j_{1}<j_{2}<\cdots<j_{m}$ (see Definition 17-(i)) and from (45).

Now, we establish the dual result of Theorem 21. For this purpose, we need the following lemma.

Lemma 22. Let $n \geq 1$ and let $u, v \in\{0,1\}^{n}$. Then $v \preceq u$ if and only if $u^{c} \leq v^{c}$. 
Proof. Clearly, the $\left(\begin{array}{l}0 \\ 0\end{array}\right),\left(\begin{array}{l}1 \\ 1\end{array}\right),\left(\begin{array}{l}0 \\ 1\end{array}\right)$, and $\left(\begin{array}{l}1 \\ 0\end{array}\right)$ columns in matrix $M_{v}^{u}$, respectively, become $\left(\begin{array}{l}1 \\ 1\end{array}\right),\left(\begin{array}{l}0 \\ 0\end{array}\right),\left(\begin{array}{l}0 \\ 1\end{array}\right)$, and $\left(\begin{array}{l}1 \\ 0\end{array}\right)$ columns in matrix $M_{u^{c}}^{v^{c}}$. Hence, using Definition 8 and Theorem 4, we have that $v \preceq u$ if and only if matrix $M_{v}^{u}$ satisfies IOC if and only if matrix $M_{u^{c}}^{v^{c}}$ satisfies IOC if and only if $u^{c} \leq v^{c}$.

Given a fixed binary $n$-tuple $u \in\{0,1\}^{n}$ with weight $m$, the following theorem provides us with the set and with the number of all the binary $n$-tuples $v \in\{0,1\}^{n}$ with weight $m$ that are intrinsically greater than or equal to $u$ (i.e., $v \geq u$ ). That is, it provides the set and the number of all the binary $n$-tuples $v$ with the same weight as $u$, and whose occurrence probabilities are always (i.e., intrinsically) greater than or equal to the occurrence probability of $u$.

Theorem 23. Let $u \in\{0,1\}^{n}, u \neq 2^{n}-1$, with (nonzero) Hamming weight $w_{H}(u)=m(0 \leq m<n)$. Let $u=$ $\left[\bar{i}_{1}, \bar{i}_{2}, \ldots, \bar{i}_{n-m}\right]_{n}^{0}$ be the vector of positions of 0 s of $u$. Then the set of the binary $n$-tuples $v$ with weight $m$, which are intrinsically greater than or equal to $u$, is given by

$$
\begin{gathered}
\left\{v \in\{0,1\}^{n} \mid w_{H}(v)=w_{H}(u), v \geq u\right\} \\
=\left\{v=\left[\bar{j}_{1}, \bar{j}_{2}, \ldots, \bar{j}_{n-m}\right]_{n}^{0} \mid \bar{j}_{1} \leq \bar{i}_{1},\right. \\
\left.j_{2} \leq \bar{i}_{2}, \ldots, \bar{j}_{n-m} \leq \bar{i}_{n-m}\right\} .
\end{gathered}
$$

Moreover, the number of the binary n-tuples $v$ with weight $m$, which are intrinsically greater than or equal to $u$, is given by

$$
\begin{gathered}
\left|\left\{v \in\{0,1\}^{n} \mid w_{H}(v)=w_{H}(u), v \geq u\right\}\right| \\
=\sum_{\bar{j}_{1}=1 \bar{j}_{2}=\bar{j}_{1}+1}^{\bar{i}_{1}} \ldots \sum_{\bar{j}_{n-m}=\bar{j}_{n-m-1}+1}^{\bar{i}_{n-m}} 1 .
\end{gathered}
$$

Proof. It suffices to use Lemma 22, Theorem 21, and the obvious fact that the 0 -bits and 1-bits in $u$ and $v$ become the 1-bits and 0-bits, respectively, in $u^{c}$ and $v^{c}$.

Example 24. Let $n=5$ and let $u=13 \equiv(0,1,1,0,1)$. Then $m=w_{H}(u)=3$.

On one hand, the vector of positions of 1-bits in $u$ is

$$
u=(0,1,1,0,1) \equiv\left[i_{1}, i_{2}, i_{3}\right]_{5}^{1}=[2,3,5]_{5}^{1} .
$$

Then, using (45) in Theorem 21, we have that the binary 5tuples $v$ with weight $m=3$, which are intrinsically less than or equal to $u=13$, are the following:

$$
\begin{aligned}
& v=[1,2,3]_{5}^{1} \equiv(1,1,1,0,0) \equiv 28, \\
& v=[1,2,4]_{5}^{1} \equiv(1,1,0,1,0) \equiv 26, \\
& v=[1,2,5]_{5}^{1} \equiv(1,1,0,0,1) \equiv 25, \\
& v=[1,3,4]_{5}^{1} \equiv(1,0,1,1,0) \equiv 22, \\
& v=[1,3,5]_{5}^{1} \equiv(1,0,1,0,1) \equiv 21,
\end{aligned}
$$

$$
\begin{aligned}
& v=[2,3,4]_{5}^{1} \equiv(0,1,1,1,0) \equiv 14, \\
& v=[2,3,5]_{5}^{1} \equiv(0,1,1,0,1) \equiv 13 .
\end{aligned}
$$

Moreover, using (46) in Theorem 21, we can confirm that the cardinality of this set is

$$
|\{13,14,21,22,25,26,28\}|=\sum_{j_{1}=1}^{i_{1}=2} \sum_{j_{2}=j_{1}+1}^{i_{2}=3} \sum_{j_{3}=j_{2}+1}^{i_{3}=5} 1=7 .
$$

On the other hand, the vector of positions of 0 -bits in $u$ is

$$
u=(0,1,1,0,1) \equiv\left[\bar{i}_{1}, \bar{i}_{2}\right]_{5}^{0}=[1,4]_{5}^{0} .
$$

Then, using (48) in Theorem 23, we have that the binary 5tuples $v$ with weight $m=3$, which are intrinsically greater than or equal to $u=13$, are the following:

$$
\begin{aligned}
& v=[1,2]_{5}^{0} \equiv(0,0,1,1,1) \equiv 7, \\
& v=[1,3]_{5}^{0} \equiv(0,1,0,1,1) \equiv 11, \\
& v=[1,4]_{5}^{0} \equiv(0,1,1,0,1) \equiv 13 .
\end{aligned}
$$

Moreover, using (49) in Theorem 23, we can confirm that the cardinality of this set is

$$
|\{7,11,13\}|=\sum_{\bar{j}_{1}=1 \bar{j}_{2}=\bar{j}_{1}+1}^{\bar{i}_{1}=1} \sum_{\bar{i}_{2}=4}^{\bar{y}_{1}} 1=3 .
$$

The following corollary characterizes the binary $n$-tuples $u$ for which the number of binary $n$-tuples $v$ intrinsically less than or equal to $u$ is maximum (minimum, resp.).

Corollary 25. Let $n \geq 1$ and $u \in\{0,1\}^{n}$ with Hamming weight $w_{H}(u)=m(0<m \leq n)$. Then

(i) the set of binary $n$-tuples $v$ with weight $m$ (the same as $u$ ) which are intrinsically less than or equal to $u$ is the set of all the $\left(\begin{array}{c}n \\ m\end{array}\right)$ binary $n$-tuples $v$ with weight $m$ if and only if the $m$ 1-bits of $u$ are placed at the $m$ right-most positions; that is, if and only if $u$ has the general pattern

$$
u=(0, \ldots, \ldots, 0,1, . ., \ldots, 1) \equiv 2^{m}-1, \quad 0<m \leq n ;
$$

(ii) the set of binary $n$-tuples $v$ with weight $m$ (the same as $u$ ) which are intrinsically less than or equal to $u$ is reduced to the n-tuple $u$ if and only if the m 1-bits of $u$ are placed at the m left-most positions; that is, if and only if $u$ has the general pattern

$$
u=(1, . \stackrel{m}{.}, 1,0, \stackrel{n-m}{\ldots}, 0) \equiv 2^{n}-2^{n-m}, \quad 0<m \leq n .
$$

Proof. (i) Let $u=\left[i_{1}, i_{2}, \ldots, i_{m}\right]_{n}^{1}$ be the vector of positions of 1 s of $u$. Using (46) in Theorem 21, we have that

$$
\begin{array}{r}
\left|\left\{v \in\{0,1\}^{n} \mid w_{H}(v)=w_{H}(u), v \leq u\right\}\right| \\
=\sum_{j_{1}=1}^{i_{1}} \sum_{j_{2}=j_{1}+1}^{i_{2}} \ldots \sum_{j_{m}=j_{m-1}+1}^{i_{m}} 1=\left(\begin{array}{c}
n \\
m
\end{array}\right)
\end{array}
$$


if and only if $i_{1}=n-m+1, i_{2}=n-m+2, \ldots, i_{m}=n$ if and only if $u$ has the pattern (56).

(ii) Using again (46) in Theorem 21, we have that

$$
\begin{gathered}
\left|\left\{v \in\{0,1\}^{n} \mid w_{H}(v)=w_{H}(u), v \preceq u\right\}\right| \\
=\sum_{j_{1}=1}^{i_{1}} \sum_{j_{2}=j_{1}+1}^{i_{2}} \ldots \sum_{j_{m}=j_{m-1}+1}^{i_{m}} 1=1
\end{gathered}
$$

if and only if $i_{1}=1, i_{2}=2, \ldots, i_{m}=m$ if and only if $u$ has the pattern (57).

Finally, we establish the dual result of Corollary 25.

The following corollary characterizes the binary $n$-tuples $u$ for which the number of binary $n$-tuples $v$ intrinsically greater than or equal to $u$ is maximum (minimum, resp.).

Corollary 26. Let $n \geq 1$ and $u \in\{0,1\}^{n}$ with Hamming weight $w_{H}(u)=m(0<m \leq n)$. Then

(i) the set of binary $n$-tuples $v$ with weight $m$ (the same as $u$ ) which are intrinsically greater than or equal to $u$ is the set of all the $\left(\begin{array}{c}n \\ m\end{array}\right)$ binary $n$-tuples $v$ with weight $m$ if and only if the $m 1$-bits of $u$ are placed at the $m$ leftmost positions; that is, if and only if $u$ has the general pattern

$$
u=(1, \ldots, \ldots, 1,0, \ldots, 0) \equiv 2^{n}-2^{n-m}, \quad 0<m \leq n
$$

(ii) the set of binary $n$-tuples $v$ with weight $m$ (the same as $u$ ) which are intrinsically less than or equal to $u$ is reduced to the $n$-tuple $u$ if and only if the $m 1$-bits of $u$ are placed at the $m$ right-most positions; that is, if and only if $u$ has the general pattern

$$
u=(0, \ldots, \stackrel{n-m}{.}, 0,1, \ldots, . \stackrel{m}{.}, 1) \equiv 2^{m}-1, \quad 0<m \leq n .
$$

Proof. Using Lemma 22 and Corollary 25, the proof is straightforward.

\section{Conclusions}

Given a fixed binary $n$-tuple $u \in\{0,1\}^{n}$, we have provided the set and the number of all the binary $n$-tuples $v$ with the same weight as $u$, and whose occurrence probabilities are always (i.e., intrinsically) less than or equal to the occurrence probability of $u$. Also, we have provided the set and the number of all the binary $n$-tuples $v$ with the same weight as $u$, and whose occurrence probabilities are always (i.e., intrinsically) greater than or equal to the occurrence probability of $u$. The special patterns of those binary strings $u$ for which the above numbers of binary strings $v$ are maximum and minimum have been identified. For future research, new relations between the intrinsic ordering and the Hamming weights of the binary $n$-tuples (arising in CSBSs) are worth to be studied.

\section{Conflict of Interests}

The author declares that there is no conflict of interests regarding the publication of this paper.

\section{Acknowledgments}

The author sincerely thanks the anonymous referee for useful comments and suggestions. This work was partially supported by the "Ministerio de Economía y Competitividad" (Spanish Government) and FEDER, through Grant Contract CGL2011-29396-C03-01.

\section{References}

[1] N. D. Singpurwalla, "Foundational issues in reliability and risk analysis," SIAM Review, vol. 30, no. 2, pp. 264-282, 1988.

[2] T. Bedford and R. M. Cooke, Probabilistic Risk Analysis: Foundations and Methods, Cambridge University Press, Cambridge, UK, 2001.

[3] M. Rausand and A. Hoyland, System Reliability Theory: Models, Statistical Methods and Applications, Wiley-Interscience, New York, NY, USA, 2004.

[4] U. S. Nuclear Regulatory Commission, "Reactor safety study: an assessment of accident risks in U. S. commercial nuclear power plants," Tech. Rep. NUREG-75/014:WASH-1400, 1975.

[5] E. Borgonovo, "The reliability importance of components and prime implicants in coherent and non-coherent systems including total-order interactions," European Journal of Operational Research, vol. 204, no. 3, pp. 485-495, 2010.

[6] C. L. Smith, "Calculating conditional core damage probabilities for nuclear power plant operations," Reliability Engineering and System Safety, vol. 59, no. 3, pp. 299-307, 1998.

[7] L. Gonzalez, "A new method for ordering binary states probabilities in reliability and risk analysis," in Computational ScienceICCS 2002, vol. 2329 of Lecture Notes in Computer Science, pp. 137-146, 2002.

[8] R. P. Stanley, Enumerative Combinatorics, vol. 1, Cambridge University Press, Cambridge, UK, 1997.

[9] L. González, "A picture for complex stochastic Boolean systems: the intrinsic order graph," in Computational Science-ICCS 2006, vol. 3993 of Lecture Notes in Computer Science, pp. 305$312,2006$.

[10] L. González, "Duality in complex stochastic Boolean systems," in Electrical Engineering and Intelligent Systems, S.-I. Ao and L. Gelman, Eds., pp. 15-27, Springer, New York, NY, USA, 2012.

[11] L. González, "Algorithm comparing binary string probabilities in complex stochastic Boolean systems using intrinsic order graph," Advances in Complex Systems, vol. 10, no. 1, pp. 111-143, 2007.

[12] L. Gonzalez, "Edges, chains, shadows, neighbors and subgraphs in the intrinsic order graph," IAENG International Journal of Applied Mathematics, vol. 42, no. 1, pp. 66-73, 2012.

[13] L. Gonzalez, "Intrinsic ordering, combinatorial numbers and reliability engineering," Applied Mathematical Modelling, vol. 37, no. 6, pp. 3944-3958, 2013. 


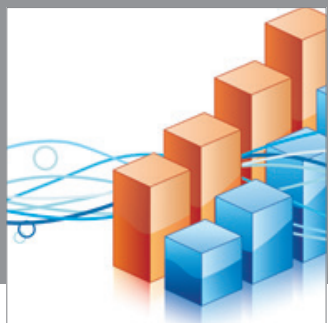

Advances in

Operations Research

mansans

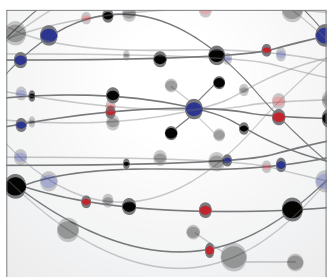

The Scientific World Journal
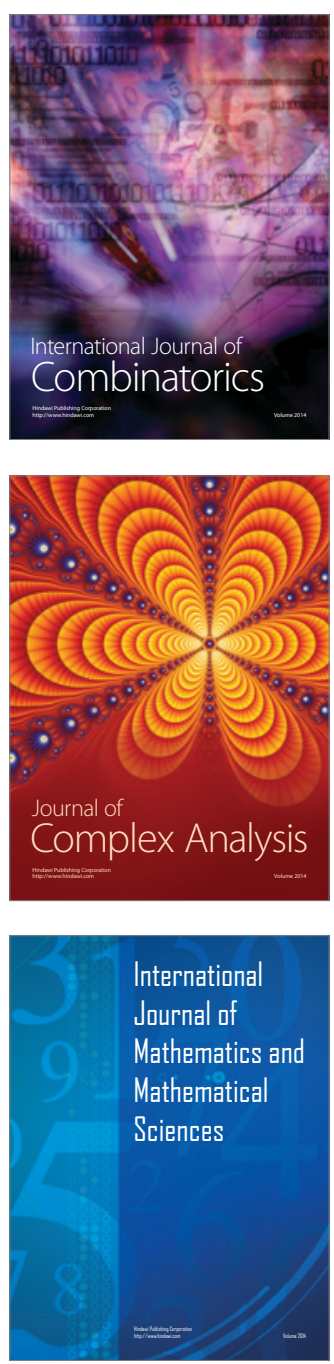
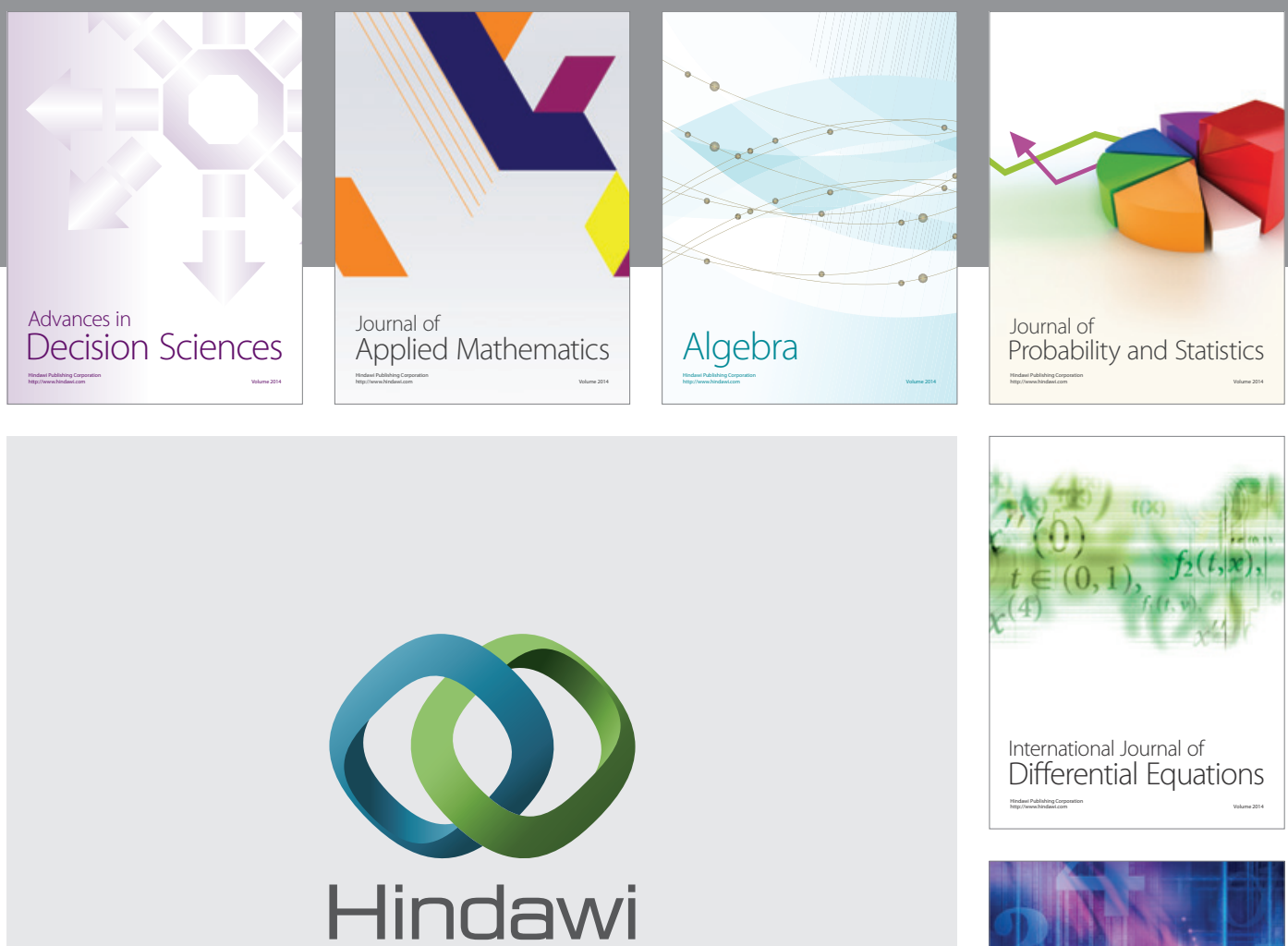

Submit your manuscripts at http://www.hindawi.com
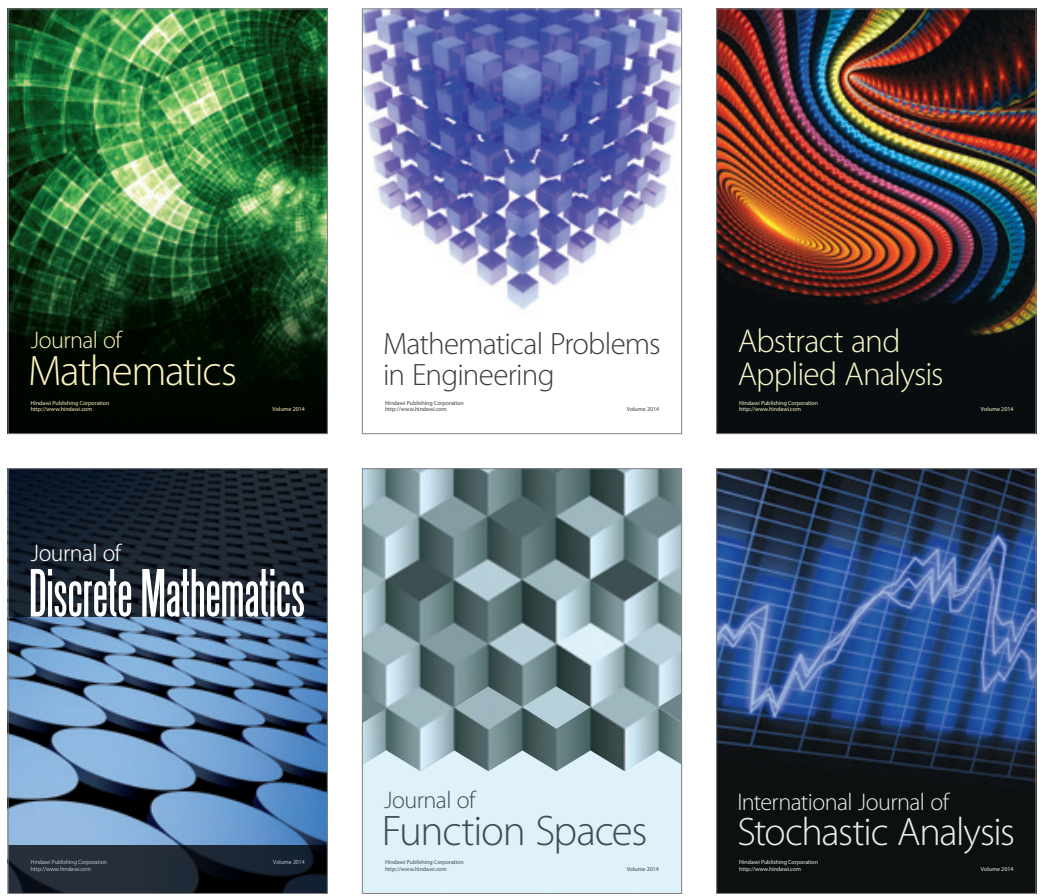

Journal of

Function Spaces

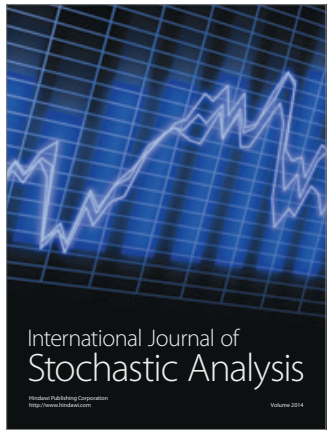

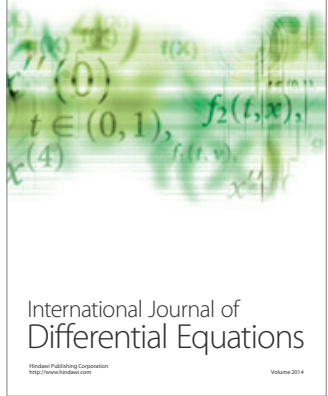
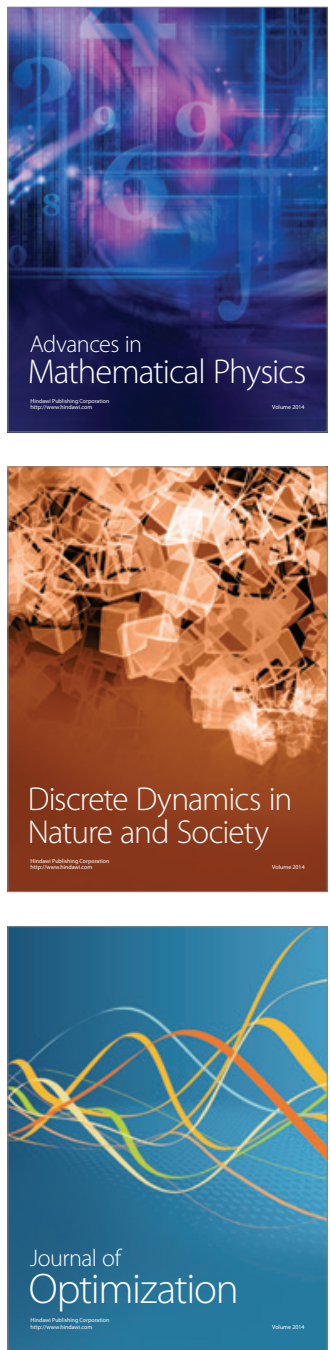